\title{
Zebrafish Models of p53 Functions
}

\author{
Narie Y. Storer and Leonard I. Zon \\ Stem Cell Program and Division of Hematology/Oncology, Children's Hospital Boston and Dana-Farber Cancer \\ Institute, Howard Hughes Medical Institute, Harvard Stem Cell Institute, Harvard Medical School, Boston, \\ Massachusetts 02115 \\ Correspondence: zon@enders.tch.harvard.edu
}

\begin{abstract}
Zebrafish models have significantly contributed to our understanding of vertebrate development and, more recently, human disease. The growing number of genetic tools available in zebrafish research has resulted in the identification of many genes involved in developmental and disease processes. In particular, studies in the zebrafish have clarified roles of the p53 tumor suppressor in the formation of specific tumor types, as well as roles of p53 family members during embryonic development. The zebrafish has also been instrumental in identifying novel mechanisms of p53 regulation and highlighting the importance of these mechanisms in vivo. This article will summarize how zebrafish models have been used to reveal numerous, important aspects of p53 function.
\end{abstract}

$T_{\text {o }}^{\text {he }}$ he zebrafish, Danio rerio, is a small model organism that has long been used to study vertebrate development. Zebrafish embryos are optically clear and develop externally to the mother, facilitating the study of early developmental processes. In addition, zebrafish have increasingly been used in modeling human diseases, including a number of cancers. The availability of forward and reverse genetic tools in the zebrafish has resulted in the identification and characterization of many genes involved in development and disease. One gene that has been extensively studied is the $p 53$ tumor suppressor gene, which is structurally and functionally conserved in the zebrafish. This article will discuss how studies in the zebrafish have increased our understanding of how p53 contributes to the formation of specific tumor types, resulted in the identification of novel mechanisms of $\mathrm{p} 53$ regulation, and showed how p53 and p53 family members are involved in embryonic development.

\section{CONSERVATION OF p53 STRUCTURE AND FUNCTION IN ZEBRAFISH}

Zebrafish p53 is highly similar to mammalian p53 in both structure and function. Full-length zebrafish p53 is $48 \%$ identical in amino acid sequence to human p53 (Cheng et al. 1997). As in mice (Rogel et al. 1985; Schmid et al. 1991), p53 is highly expressed early in zebrafish development. p53 transcript is most highly expressed in embryos within $1 \mathrm{~h}$ postfertilization (hpf) (Cheng et al. 1997) and is ubiquitously expressed in the early embryo (Thisse

Editors: Arnold J. Levine and David P. Lane

Additional Perspectives on The p53 Family available at www.cshperspectives.org

Copyright (C) 2010 Cold Spring Harbor Laboratory Press; all rights reserved; doi: 10.1101/cshperspect.a001123

Cite this article as Cold Spring Harb Perspect Biol 2010;2:a001123 
et al. 2000). At the pharyngula stage (24-48 hpf), p53 is predominantly expressed in the head region. By 48 hpf, p53 transcript and protein levels are barely detectable (Cheng et al. 1997; Thisse et al. 2000; Lee et al. 2008).

To elucidate p53 function in zebrafish, Langheinrich and colleagues injected zebrafish embryos with p53 antisense morpholinos that knockdown p53 expression in the embryo (Langheinrich et al. 2002). The morpholinoinjected, or morphant, embryos are developmentally normal. However, as compared with wild-type embryos, the p53 morphants displayed a decreased induction of apoptosis on DNA-damaging stimuli, including UV irradiation and the topoisomerase inhibitor camptothecin. Studies in zebrafish that harbor a missense mutation, $\mathrm{M} 214 \mathrm{~K}$, in the DNAbinding domain of p53 have yielded similar results (Berghmans et al. 2005). Although developmentally normal, p53 mutant embryos fail to undergo apoptosis on $\gamma$-irradiation. Moreover, a growing body of evidence suggests that in zebrafish, p53 regulates apoptosis in response to a myriad of stimuli (Langheinrich et al. 2002; Berghmans et al. 2005; Robu et al. 2007; Lee et al. 2008). Thus, as in mammals and other model organisms, p53 is a key mediator of apoptosis in the zebrafish.

Other aspects of p53 regulation and function are also conserved in zebrafish. The major functional domains of p53 negative regulator MDM2, and the physical interaction between p53 and MDM2, are highly conserved in zebrafish (Thisse et al. 2000). MDM2-deficient zebrafish embryos display growth retardation and high levels of apoptosis. These embryos are completely rescued by p53 deficiency (Langheinrich et al. 2002), akin to observations in $p 53 / M d m 2$ double knockout mice (Jones et al. 1995; Montes de Oca Luna et al. 1995). However, recent data suggest that the apoptosis seen in MDM2-deficient embryos may be because of off-target effects of the MDM2 morpholino (see following discussion) (Robu et al. 2007), so further experiments will be required to elucidate the interaction of MDM2 and p53 in zebrafish embryos. In addition, zebrafish p53 is required for transcriptional regulation of a number of known p53 target genes, including bax, $m d m 2$, and $p 21$ (Langheinrich et al. 2002; Lee et al. 2008). Lastly, p53 mutant embryos fail to undergo G1 arrest on $\gamma$-irradiation, suggesting a conserved role for zebrafish p53 in cell cycle regulation (Berghmans et al. 2005). As discussed later, studies on p53 in the zebrafish have also revealed novel aspects of p53 function that are important in the function of p53 in higher vertebrates.

\section{p53 IN ZEBRAFISH MODELS OF CANCER}

\section{Tumor Formation in p53 Mutant Zebrafish}

The importance of $p 53$ in cancer is highlighted by the fact that it is the most frequently mutated tumor suppressor gene in human cancers, with more than half of solid tumors harboring mutations in the $p 53$ locus. In many other tumors, p53 is inactivated by more indirect mechanisms, including MDM2 amplification and p14ARF deletion (Vogelstein et al. 2000). Patients with Li-Fraumeni syndrome, characterized by germline inactivating mutations in $p 53$, have a significantly increased risk of developing cancer (Kleihues et al. 1997; Nichols et al. 2001). Moreover, mice homozygous for a $p 53$ null allele also have an increased propensity to develop tumors (Donehower et al. 1992; Jacks et al. 1994).

p53 also acts as a tumor suppressor in zebrafish. Fish homozygous for the $p 53^{M 214 K}$ mutation develop tumors at $28 \%$ incidence by 16.5 mo of life, whereas wild-type and heterozygous fish seldom develop tumors. Histologically, these tumors most closely resemble human malignant peripheral neural sheath tumors (MPNSTs). The zebrafish MPNSTs displayed morphological features of nerve sheath differentiation by electron microscopy and expressed the neuronal marker $g f a p$. The tumors were also clonally aneuploid and had a long latency, suggesting that additional mutations are required for the initiation of zebrafish MPNSTs (Berghmans et al. 2005). Although almost all tumors that developed in $p 53^{M 214 K}$ zebrafish were characterized as a MPNST, humans and mice with germline mutations of $p 53$ do not commonly develop MPNSTs (Donehower et al. 1992; Jacks 
et al. 1994; Kleihues et al. 1997; Nichols et al. 2001). The reasons for this difference in tumor spectrum are unclear, but they are likely attributable to species- and tissue-specific differences in the response to DNA damage and unchecked proliferative stimuli (Berghmans et al. 2005).

As in zebrafish, $p 53$ mutations are likely to be an important contributor to the development of mammalian MPNSTs. Nf1 knockout mice develop benign nerve sheath tumors, whereas Nf1;p53 double knockout mice form MPNSTs, indicating that $p 53$ mutations cooperate with $N f 1$ deficiency in the development of murine MPNSTs (Cichowski et al. 1999; Vogel et al. 1999). In addition, deletions and mutations in the $p 53$ locus (Menon et al. 1990) and deletions in the CDKN2A/p16 locus (Nielsen et al. 1999) have been identified in human MPNSTs, suggesting a role for p53 pathway disruption in human MPNST. Thus, p53 mutant zebrafish can be a useful model in elucidating how p53 pathway dysregulation contributes to MPNSTs and identifying other pathways that contribute to the pathogenesis of MPNSTs. Moreover, as discussed in the following sections, the $p 53$ mutant zebrafish has proved useful in clarifying the role of p53 mutations in the formation of other tumor types.

\section{Cooperativity of p53 and BRAF Mutations in Zebrafish Melanoma}

The majority of human melanomas harbor activating mutations in BRAF, an effector of RAS/MAPK signaling that mediates cellular proliferation and other processes (Brose et al. 2002; Davies et al. 2002; Tuveson et al. 2003). Activating BRAF mutations are also commonly observed in benign nevi (Pollock et al. 2003), or moles, suggesting that mutant $B R A F$ cooperates with other mutations to induce melanoma formation. To investigate the effect of activated BRAF on melanoma development, Patton and colleagues generated a transgenic construct in which activated human $B R A F^{V 600 E}$ is expressed under the control of the zebrafish melanocyte lineage-specific mitfa promoter (Patton et al. 2005). The mitfa-BRAF ${ }^{V 600 E}$ transgene was microinjected into zebrafish embryos, and resulting mosaic transgenic fish developed focal areas of melanocyte proliferation, termed fish nevi (f-nevi), that resemble their human counterparts. The f-nevi were characterized by an increased number of well-differentiated melanocytes without features of dysplasia or invasion into neighboring tissues. Stable transgenic mitfa-BRAF ${ }^{V 600 E}$ fish that express the transgene in all melanocytes display abnormal stripe patterning. mitfa-BRAF ${ }^{V 600 E}$ fish do not develop melanoma, suggesting that, whereas activated BRAF is sufficient to induce melanocyte proliferation, additional mutations are required for the progression to malignant melanoma (Patton et al. 2005).

Multiple lines of evidence have suggested that disruption of $\mathrm{p} 53$ pathway activity contributes to melanoma formation. $p 53$ mutations are found in $10 \%-25 \%$ of human melanomas (Daniotti et al. 2004; Ceol et al. 2008). In addition, a larger proportion of melanomas harbor mutations that inactivate the CDKN2A locus, which encodes two distinct tumor suppressor genes, INK4A and ARF. The ARF tumor suppressor inhibits MDM2-mediated ubiquitylation and the ensuing degradation of p53. Thus, mutations in the CDKN2A locus commonly found in melanoma often result in the disruption of p53 activity. Furthermore, p53 pathway activity, as assessed by gene expression changes in response to DNA damage, is attenuated in many melanomas (Kaufmann et al. 2008). However, the requirement of p53 pathway disruption in the formation of melanoma has been unclear.

To investigate the contribution of p53 disruption to melanoma formation, the mitfa$B R A F^{V 600 E}$ construct was microinjected into $p 53^{M 214 K}$ mutant zebrafish. Interestingly, half of fish that developed nevi also formed melanoma by 4 mo of age. These tumors resembled human melanoma by histology. In addition, the tumors were highly invasive, transplantable into recipient fish, and often showed aneuploidy. In contrast, melanoma formation is much more rare in wild-type and $p 53^{M 214 K}$ zebrafish. Thus, p53 mutation cooperates with BRAF activation in the formation of zebrafish melanoma (Patton et al. 2005). This study importantly showed 
that activating BRAF mutations and p53 pathway mutations participate in the formation of melanomas and are not merely bystander mutations formed during tumor development (Ceol et al. 2008). In addition, this zebrafish model can be useful in understanding how p53 loss-of-function contributes to melanoma pathogenesis.

Cooperativity of p53 and RAS Mutations in Zebrafish Rhabdomyosarcoma

Rhabdomyosarcoma (RMS) is a malignancy with characteristics of muscle differentiation and is the most common soft-tissue sarcoma of childhood (Arndt and Crist 1999). Embryonal rhabdomyosarcoma (ERMS) is the most prevalent subtype of pediatric RMS and is characterized in a minority of cases by mutational activation of RAS family members (Stratton et al. 1989; Chen et al. 2006). Zebrafish embryos were injected with a transgenic construct in which human oncogenic KRAS ${ }^{G 12 D}$ is expressed under the control of a fragment of the zebrafish rag 2 promoter that drives gene expression in satellite cells and myoblasts within skeletal muscle (Langenau et al. 2007). The resulting rag2-KRAS ${ }^{G 12 D}$ mosaic transgenic zebrafish began to develop tumors by $10 \mathrm{dpf}$ that resembled ERMS by histology and were highly invasive. Gene set enrichment analysis of the most up-regulated genes in the zebrafish tumors revealed that the tumors were highly similar to human ERMS by gene expression. Hence, expression of activated KRAS in muscle progenitor cells is sufficient to induce ERMS formation in zebrafish (Langenau et al. 2007).

p53 mutations are observed in a subset of ERMS cases (Xia et al. 2002), suggesting that inactivation of the $\mathrm{p} 53$ pathway can contribute to the pathogenesis of ERMS. Sequencing of the $p 53$ locus in zebrafish ERMS tumors failed to identify inactivating mutations in $p 53$. However, $m d m 2$ and survivin, negative regulators of p53 pathway activity, are highly expressed in the zebrafish tumors, indicating that the p53 pathway may be disrupted in zebrafish ERMS (Langenau et al. 2007). Overexpression of MDM2 and SURVIVIN is also observed in human RMS
(Xia et al. 2002; Caldas et al. 2006). Interestingly, homozygous and heterozygous $p 53^{M 214 K}$ mutants injected with rag2-KRAS ${ }^{G 12 D}$ have a significantly increased tumor incidence in comparison to wild-type siblings, demonstrating that p53 pathway disruption can cooperate with RAS mutations in the formation of ERMS (Langenau et al. 2007). These data suggest that inactivation of the p53 pathway is important in ERMS tumor initiation in both zebrafish and humans.

\section{REGULATION OF p53 IN ZEBRAFISH}

p53 plays important roles in controlling cell cycle progression, cellular senescence and apoptosis in response to various cellular stressors. Thus, tight regulation of p53 activity is required for normal cell proliferation and the prevention of tumor formation, and disruption in this regulation is a commonly observed mechanism of p53 pathway inactivation in human cancer. In mammals, p53 stability and function is regulated by a number of posttranslation modifications (Brooks and Gu 2003), whereas in zebrafish, regulation at both the mRNA and protein levels in response to different types of stress has been described (Langheinrich et al. 2002; Lee et al. 2008). Recent studies in zebrafish have highlighted novel and varied ways in which p53 can be regulated during normal development and tumorigenesis.

\section{Delta113p53 Regulation of p53-Mediated Apoptosis}

A number of p53 isoforms generated by alternative splicing and promoter usage have been identified in human cells (Bourdon et al. 2005). One such isoform, $\Delta 133 p 53$, and its zebrafish ortholog, $\Delta 113 p 53$, are transcribed from an alternative promoter in intron 4 of $p 53$ and lack the amino-terminal transactivation domain and part of the DNA binding domain of p53 (Bourdon et al. 2005; Chen et al. 2005). Because the $\Delta 133 p 53$ and $\Delta 113 p 53$ isoforms lack the transactivation domain but have a largely intact DNA binding domain, these isoforms are postulated to have a dominant-negative effect 
in the regulation of p53 transcriptional activity. In concordance with this hypothesis, cotransfection of $\Delta 133 p 53$ with $p 53$ in human cells inhibits p53-induced apoptosis (Bourdon et al. 2005).

The role of this p53 isoform in regulating p53 has been further clarified in the zebrafish (Chen et al. 2005; Chen et al. 2009). The zebrafish $\Delta 113 p 53$ promoter has a number of p53 consensus binding sites that are required for transcriptional activity and is directly regulated by $\mathrm{p} 53$. $\Delta 113 p 53$ is up-regulated in zebrafish embryos on treatment with DNA-damaging agents in a p53-dependent manner. As in human cells, overexpression of $\Delta 113 p 53$ rescues p53-induced apoptosis in zebrafish embryos. Moreover, morpholino knockdown of $\Delta 113$ p53 strongly enhances p53-mediated apoptosis in response to ionizing radiation treatment. These data suggest that $\Delta 113 p 53$ expression has a protective effect against p53-mediated apoptosis in zebrafish (Chen et al. 2009).

Interestingly, $\Delta 113$ p53 has varying effects on the transcription of p53-regulated genes in zebrafish. $\Delta 113$ p53 enhances p53-dependent up-regulation of $p 21$ and $m d m 2$, but inhibits p53-dependent up-regulation of the proapoptotic gene bax. In contrast, whereas overexpression of $p 53$ results in down-regulation of the antiapoptotic gene $b c l 2 L$, co-overexpression of $\Delta 113 p 53$ restores $b c l 2 L$ expression. In fact, $\Delta 113 p 53$ requires $b c l 2 L$ expression to inhibit p53-mediated apoptosis. Thus, $\Delta 113 p 53$ does not act simply in a dominant negative fashion to inhibit p53 function, but rather modulates p53-dependent transcription in a target-specific manner (Chen et al. 2009).

Studies in zebrafish also suggest that $\Delta 113 p 53$ plays a role in vertebrate development (Chen et al. 2005; Chen et al. 2009). A loss-offunction mutation in def (digestive-organ expansion factor) results in the formation of hypoplastic digestive organs because of cell cycle arrest, rather than apoptosis, in these organs. Mutant embryos also display specific up-regulation of $\Delta 113 p 53$, but not full-length $p 53$, in the digestive tract in zebrafish embryos. Although knockdown of both $\Delta 113 p 53$ and $p 53$ partially rescued the mutant phenotype (Chen et al. 2005), knockdown of $\Delta 113 p 53$ alone enhances apoptosis in the digestive organs of def morphants (Chen et al. 2009). Interestingly, cell cycle-related, but not proapoptotic, p53 target genes are up-regulated in def mutant embryos (Chen et al. 2005). Thus, studies in zebrafish have shown that $\Delta 113 p 53$ functions to regulate p53 activity during development, as well as in response to DNA-damaging agents.

\section{Regulation by Ribosomal Proteins}

A large-scale insertional mutagenesis screen in the zebrafish identified more than 300 genes, including 28 ribosomal protein $(r p)$ genes, required for early development (Amsterdam et al. 1999; Golling et al. 2002; Amsterdam et al. 2004a). Although homozygous $r p$ mutants are embryonic lethals, heterozygous $r p$ mutant fish are viable through adulthood. Fish with heterozygous mutations in 17 of the 28 rp genes develop malignant peripheral nerve sheath tumors (MPNSTs) with 10\%-100\% incidence (Amsterdam et al. 2004b; Lai et al. 2009). Interestingly, whereas MPNSTs are exceedingly rare $(0.05 \%)$ in wild-type zebrafish (Lai et al. 2009), 28\% of p53 homozygous mutant fish develop MPNSTs by 16.5 months of life (Berghmans et al. 2005). In addition, MPNSTs from $r p^{+/-}$and $p 53^{-/-}$mutants have very similar gene expression profiles (MacInnes et al. 2008). Collectively, these data hinted at a role for $r p$ heterozygosity in p53 pathway regulation.

A closer examination of $r p^{+/-}$tumors revealed that tumors expressed wild-type $p 53$ mRNA at detectable levels. However, MPNSTs from all $17 r p^{+/-}$lines expressed little or no p53 protein, whereas $p 53^{-/}$MPNSTs express high levels of mutant $\mathrm{p} 53$. Moreover, $r p^{+/-}$ MPNSTs do not display a p53-dependent induction of p53 mRNA expression on $\gamma$-irradiation, suggesting a loss of p53 protein function in these tumors. p53 protein levels remain low in $r p^{+/-}$MPNSTs even after exposure to $\gamma$-irradiation or the proteasome inhibitor MG132, indicating that the low p53 protein levels observed are not because of enhanced protein degradation. Although overall translation capacity is normal in $r p^{+/-}$MPNSTs, 
p53 protein synthesis is specifically impaired in the tumors (MacInnes et al. 2008). These observations show that $r p$ heterozygosity specifically impairs translation of $p 53$ mRNA and highlight a novel mechanism of $\mathrm{p} 53$ regulation. Because this effect was observed for all $17 r p$ genes studied, it is likely that p53 regulation in these lines occurs by a common mechanism, perhaps by deficiency in ribosomal function. Furthermore, these data also suggest a role for $r p$ gene dosage in tumorigenesis.

Although this effect on $\mathrm{p} 53$ protein levels was seen in 17 different $r p$ lines, down-regulation of p53 by rp deficiency is not universally observed. First, p53 protein loss was only observed in tumor cells, and not in all cells, in the 17 $r p^{+/-}$lines that were studied (MacInnes et al. 2008). Thus, defects in p53 synthesis may be tumor- or tissue-specific. In addition, homozygous mutant embryos for three of the 17 rp lines display p53 pathway activation (Danilova et al. 2008). Similarly, injection of morpholinos for two other $r p$ genes results in activation of p53 target genes (Danilova et al. 2008; Chakraborty et al. 2009). Hence, a complete or near complete loss of $r p$ expression does not inhibit p53 function, but rather can result in p53-dependent apoptosis (Chakraborty et al. 2009). Zebrafish embryos with deficiencies in rRNA synthesis and processing, and presumably ribosome biogenesis, also activate the p53 pathway (Azuma et al. 2006; Skarie and Link 2008). Thus, regulation of p53 by rps may depend on cellular context, rp expression level, and level of ribosomal function. This work has revealed an important role of rps in the specific regulation of $\mathrm{p} 53$, and deficiency in rps may be a mechanism of p53 dysregulation in human tumors.

\section{Regulation by MicroRNA-125b}

MicroRNAs (miRNAs) are a large group of small, noncoding RNAs that regulate gene expression by mRNA cleavage or translational repression, largely through binding with partial complementarity to the $3^{\prime}$ untranslated region (UTR) of target mRNAs (Bartel 2004). miRNAs have been increasingly implicated in a number of biological and disease processes, including cancer (Esquela-Kerscher and Slack 2006). Recent work has shown that a miRNA, miR-125b, directly regulates p53 in zebrafish and in human cells (Le et al. 2009). miR-125b is able to bind to the $3^{\prime}$ UTR of both zebrafish and human p53 mRNA and negatively regulates zebrafish and human p53 protein levels when ectopically expressed. This regulation requires an intact, predicted miR-125b binding site in the zebrafish and human $3^{\prime}$ UTR. In addition, miR125 b negatively regulates endogenous p53 protein levels and levels of apoptosis in human neuroblastoma cells and in primary human lung fibroblasts (Le et al. 2009).

The regulation of p53 by miR-125b is also important during development. Expression of miR-125b in the developing zebrafish embryo begins at $19 \mathrm{hpf}$. It is expressed throughout the embryo, but is enriched in the brain, eyes, and somites. Interestingly, there is an inverse correlation between miR-125b expression levels and $p 53$ or p21 expression levels in the developing embryo. Embryos injected with morpholinos against miR-125b display neural death specific to miR-125b knockdown and morphological defects including loss of the midbrainhindbrain boundary and deformed somites. Morphant embryos have elevated levels of p53 protein and p21 mRNA, suggesting p53 pathway activation in embryos on miR-125b loss. In addition, morphant embryos displayed elevated neural apoptosis by TUNEL assay that was rescued by overexpression of a miR-125b duplex. The neural apoptosis and many of the morphological defects could be rescued by p53 loss-of-function, suggesting that the p53 pathway is the major target of miR-125b during development (Le et al. 2009).

As mentioned earlier, zebrafish embryos undergo p53-mediated apoptosis on exposure to DNA-damaging agents, such as $\gamma$-irradation and camptothecin. Notably, $\boldsymbol{\gamma}$-irradiation or camptothecin treatment results in a significant down-regulation of miR-125b expression and concomitant increase in p53 protein levels in zebrafish embryos. Ectopic expression of miR$125 \mathrm{~b}$ in these embryos substantially decreases p53 levels and rescues stress-induced apoptosis. Moreover, treatment of human neuroblastoma 
cells with etoposide, a topoisomerase inhibitor that induces DNA damage and activates p53, results in a down-regulation of miR-125b levels. Thus, down-regulation of miR-125b may be a conserved mechanism to activate p53 and subsequent p53-mediated apoptosis in response to DNA damage and perhaps other cellular stressors (Le et al. 2009).

In summary, the direct regulation of p53 protein levels by miR-125b plays important roles during development and in the regulation of stress-induced apoptosis. In addition, recent data also suggest that upstream regulators of p53 may also be targets of miR-125b (Sinha et al. 2008). Hence, miR-125b is likely to play multiple, important roles in regulating the p53 pathway. Given its importance as a negative regulator of p53, it will be intriguing to evaluate the oncogenic potential of $\mathrm{miR}-125 \mathrm{~b}$ and its role in p53 regulation in tumors.

\section{ROLE OF p53 IN MORPHOLINO TOXICITY}

Morpholino antisense oligonucleotides and short interfering RNA (siRNA) are commonly used reagents to elicit gene-specific knockdown in a variety of systems. However, a major limitation of both technologies is the induction of off-target effects (Ekker and Larson 2001; Jackson and Linsley 2004). In particular, injection of morpholinos into zebrafish embryos often results in a characteristic neural death by 22 hpf (Ekker and Larson 2001). This phenotype is not specific to the genes targeted for knockdown, as available loss-of-function mutations in corresponding genes do not elicit this neural death. Also, the off-target effect is morpholino sequence-specific - that is, unique morpholinos targeting different sequences in the same gene may or may not elicit an offtarget effect (Robu et al. 2007).

Closer examination of Smo and Wnt 5 morphant embryos displaying the off-target neural phenotype revealed $p 21$ up-regulation and extensive neural apoptosis by TUNEL assay from 14 to $26 \mathrm{hpf}$, suggesting p53 activation in these embryos. Simultaneous morpholino knockdown of p53 in these embryos rescued the neural apoptosis without affecting gene-specific phenotypes. Importantly, p53 knockdown does not affect gene-specific apoptosis in the tails of chordin morphants, and does not affect genespecific phenotypes in nacre, no tail and UROD morphants. p53 coknockdown is also able to distinguish craniofacial defects that are gene knockdown-specific from those that are the result of off-target effects (Robu et al. 2007).

These results highlight the importance of discerning gene-specific versus off-target effects in morpholino knockdown experiments (Robu et al. 2007). Coknockdown of p53 can distinguish off-target effects, although caution is warranted as some morphant phenotypes may be the result of gene-specific activation of the p53 pathway. Other experiments can also aid in identifying knockdown phenotypes that are gene-specific. Effects are likely gene-specific if they can be rescued by DNA/RNA of the gene of interest, although such rescue experiments can often be difficult to interpret. Moreover, effects that are elicited with multiple, different morpholino sequences targeting the same gene are also likely to be gene-specific. Finally, nonspecific activation of p53 has been described in siRNA-treated cells (Scacheri et al. 2004), suggesting that inhibition of p53 may be effective in reducing the off-target effects of other knockdown technologies (Robu et al. 2007).

\section{p53 FAMILY MEMBERS IN DEVELOPMENT}

Although p53 plays a critical role in cell cycle control and the regulation of apoptosis, it is largely dispensable for normal embryonic development in fish and mice. $p 53^{M 214 K}$ mutant zebrafish embryos and p53 morphant embryos develop normally and are morphologically indistinguishable from wild-type embryos (Langheinrich et al. 2002; Berghmans et al. 2005). In addition, heterozygous and homozygous $p 53^{M 214 K}$ mutant fish display normal viability, yet also show a decreased fecundity, in comparison to wild-type siblings (Langheinrich et al. 2002). Similarly, the majority of mice homozygous for a $p 53$ null allele appear developmentally normal by morphology and histology (Donehower et al. 1992). However, a minority of homozygous mutant mice displays 
exencephaly and defects in neural tube closure, phenotypes predominantly associated with female embryos (Armstrong et al. 1995; Sah et al. 1995). The penetrance of these defects varies with genetic background, suggesting that other modifying genes can interact with $p 53$ mutations to affect neural tube closure (Sah et al. 1995). Collectively, these data indicate that, in general, p53 is not essential for normal embryonic development.

In contrast, studies in zebrafish have shown that p53 family members, p63 and p73, are required in a number of processes in the developing embryo. $p 63$ and $p 73$ are each transcribed from two different promoters and are alternatively spliced to generate gene products with differing carboxy-termini (Yang et al. 1998; Romani et al. 2003). Transcription from an upstream promoter results in TA isoforms that, like full-length p53, contain a amino-terminal transactivation domain, a DNA binding domain, and an oligomerization domain, whereas transcription from a more downstream site results in amino-terminally $(\Delta \mathrm{N})$ truncated isoforms that lack the transactivation domain. Because the $\Delta \mathrm{N}$ isoforms lack a transactivation domain, they are thought to act in a dominant-negative fashion to inhibit the normal transcriptional activity of full-length p53 family members. Studies in the zebrafish have highlighted the importance of TA and $\Delta \mathrm{N}$ isoforms of p63 and p73 during embryonic development and have suggested a role for these isoforms in modulating p53 activity in vivo.

\section{The Role of $\Delta \mathrm{Np} 63$ in Ectodermal Development}

p63 is required for the normal development of ectoderm-derived tissues. Knockout mice lacking both TAp63 and $\Delta \mathrm{Np} 63$ isoforms fail to develop stratified squamous epithelia and their derivatives, including skin and hair, and do not form limbs (Mills et al. 1999; Yang et al. 1999). In addition, patients harboring p63 mutations display a number of skin and limb defects (Celli et al. 1999; McGrath et al. 2001). However, it was unclear from these studies whether loss of TAp63 and/or $\Delta \mathrm{Np} 63$ isoforms contributed to these defects and how loss of p63 function affected specific stages of ectodermal development.

Studies in zebrafish have clarified the roles of p63 isoforms during development. $\Delta$ Np63 isoforms are conserved in the zebrafish and are the predominant isoforms expressed during embryonic development (Bakkers et al. 2002; Lee and Kimelman 2002). $\Delta N p 63$ is expressed in the ventral ectoderm during gastrulation and is later maintained in ectoderm-derived tissues. Embryos injected with high levels of morpholino specifically targeting $\Delta \mathrm{Np} 63$, and not TAp63, isoforms show a decrease in nonneural (ventral, epidermal) ectoderm and an expansion of dorsal neural ectoderm at mid-gastrula stages, indicating that $\Delta \mathrm{Np} 63$ is required for proper dorsoventral (DV) patterning. Interestingly, this effect was phenocopied by overexpression of TA forms of p53 family members. Conversely, overexpression of $\Delta N p 63$ in embryos resulted in an expansion of nonneural ectoderm at the expense of anterior neural structures, which could be rescued by overexpression of murine TAp63. $\Delta$ Np63 was shown to be a direct transcriptional target of Bmp signaling and to function downstream of Bmp signaling in inhibiting neural cell fates in the ventral ectoderm (Bakkers et al. 2002).

$\Delta$ Np63 also functions later in the development of ectoderm-derived tissues. Notably, whereas $\Delta \mathrm{Np} 63$ protein is present throughout cells during early development, it becomes restricted to the nuclei of the epidermis and eye epithelium at $20 \mathrm{hpf}$, a stage when fin folds begin to protrude from the embryo because of epidermal expansion (Lee and Kimelman 2002). Embryos injected with $\Delta$ Np63-specific morpholinos lack pectoral fins, likely because of a failure to maintain the apical fold (Bakkers et al. 2002; Lee and Kimelman 2002). The apical fold of fin buds in zebrafish is homologous to the apical ectodermal ridge (AER) of higher vertebrates, an epidermal structure at the distal end of limb buds that is required for vertebrate limb development ( $\mathrm{Ng}$ et al. 1999). Morphant embryos also display skin lesions by 3dpf (Bakkers et al. 2002; Lee and Kimelman 2002). Although $\Delta$ Np63 marks BrdU-positive, 
proliferating epithelial cells in wild-type zebrafish embryos, $\Delta$ Np63-morphant embryos lose all BrdU-positive cells in the epidermis, indicating that $\Delta \mathrm{Np} 63$ is absolutely required for the proliferation of epidermal cells. This effect of $\Delta$ Np63 on epidermal proliferation in zebrafish may be because of the inhibition of p53 activity by $\Delta \mathrm{Np} 63$. Moreover, induced p53 overexpression in embryos results in fin and skin defects akin to those observed in $\Delta \mathrm{Np} 63$ morphants, and these defects are rescued by overexpression of an inducible $\Delta$ Np63 (Lee and Kimelman 2002).

These studies using the zebrafish show specific roles for the $\Delta \mathrm{N}$ isoform of p63 in both DV patterning of the ectoderm and epidermal proliferation during development. Both roles of $\Delta$ Np63 appear to be at least partially mediated by inhibition of the activity of p53 family members during development. Interestingly, zebrafish $\Delta \mathrm{Np} 63$ can act as a dominant negative inhibitor of p53 transcriptional activation in both tissue culture and in zebrafish embryos (Lee and Kimelman 2002). Future experiments will likely elucidate how modulation of the transcriptional activity of p53 family members by $\Delta \mathrm{Np} 63$ is involved in the proper development of ectoderm-derived tissues.

\section{TAp73 is Required for Normal Embryonic Development}

Like p63, p73 is also essential for normal embryonic development. In mice, $\Delta \mathrm{Np} 73 \alpha$ is the major isoform expressed in embryos and adults, and can act in a dominant negative fashion to inhibit TAp73-mediated transcriptional activation. Murine p73 is expressed in the nasal epithelium, hippocampus and hypothalamus in developing embryos. p73-deficient mice that lack all p73 isoforms display defects in neuronal development and pheromone sensing, and also show hydrocephalus and chronic inflammation (Yang et al. 2000). $\Delta$ Np73 isoforms have an antiapoptotic, prosurvival role in at least some neurons of the central and peripheral nervous systems during neural development and are also important for the long-term maintenance of adult neurons (Pozniak et al. 2000; Pozniak et al. 2002).

In contrast to the mouse, $\operatorname{TAp} 73 \alpha$ is the major p73 isoform expressed in zebrafish embryos, and $\Delta \mathrm{N}$ isoforms have so far been undetectable in zebrafish (Pan et al. 2003; Rentzsch et al. 2003). TAp73 transcript is detected in zebrafish embryos as early as mid-gastrulation, and is expressed in the developing olfactory system and telencephalon, similar to observations in mouse embryos (Yang et al. 2000). In addition, $p 73$ expression can be seen in the pharyngeal endoderm, pronephric ducts and slow muscle cells of developing zebrafish, but not mouse, embryos (Yang et al. 2000; Rentzsch et al. 2003). Zebrafish embryos injected with morpholinos targeting p73 show defects in the formation of the olfactory system and telencephalon, reminiscent of defects in the olfactory system and neurons in p73-knockout mice. In addition, p73 morphant embryos show craniofacial malformations; whereas all cartilage elements are present, they are generally misshapen in the morphants (Rentzsch et al. 2003). This phenotype is specific to zebrafish, as craniofacial development is not affected in p73 null mice (Yang et al. 2000).

Like transcriptionally active forms of other p53 family members, zebrafish TAp73 can antagonize the activity of $\Delta \mathrm{Np} 63$ overexpression in embryos, suggesting that TAp73 shares some of the transactivating activities of the p53 family. However, whereas overexpression of p53 results in elevated levels of apoptosis in zebrafish embryos, overexpression of TAp73 does not have a proapoptotic effect. In addition, morpholino-induced knockdown of TAp73, unlike knockdown of p53, does not prevent apoptosis induced by UV irradiation (Rentzsch et al. 2003). In contrast, in mammalian cell culture, TAp73 can induce apoptosis through the regulation of p53-responsive genes (Jost et al. 1997). This difference in the regulation of apoptosis may be attributable to differences in the target genes of TAp73 in mice and fish.

$\Delta \mathrm{N}$ and TA isoforms of p53 family members are generally thought to have antagonizing activities. Although different p73 isoforms are expressed in mice and fish during embryonic 
N.Y. Storer and L.I. Zon

development ( $\Delta \mathrm{N}$ and TA, respectively), deficiency of p73 in both species results in similar defects in the development of the telencephalon and olfactory system. Thus, it is possible that the $\Delta \mathrm{N}$ and TA isoforms of p73 have some shared activity, perhaps in the regulation of transcriptional targets. It will be interesting to discern the overlapping and distinct functions of $\Delta \mathrm{N}$ and TA isoforms of p73 during embryonic development.

\section{CONCLUDING REMARKS}

The $\mathrm{p} 53$ tumor suppressor is a master regulator of cell cycle progression and apoptosis in response to cellular stressors. The significance of p53 in regulating cellular proliferation is underscored by the fact that it is mutated in more than half of all human solid tumors. Studies in zebrafish have shown the importance of p53 mutations in various tumor types and have clarified how $\mathrm{p} 53$ and p 53 family members function during embryogenesis. The zebrafish has also been instrumental in identifying novel mechanisms of p53 regulation that may contribute to p53 pathway disruption in human tumors. The ease of performing forward and reverse genetic techniques and chemical screens make the zebrafish ideally suited for the future identification and characterization of genes and drugs that modify p53 pathway activity in vertebrates.

\section{ACKNOWLEDGMENTS}

We thank Craig Ceol and Gessingga Storer for critical reading of this manuscript. N.Y.S. was supported in part by National Institutes of Health (NIH) Cell and Developmental Biology Training Grant GM07226. Cancer research by L.I.Z. is funded by NIH Grant 5R01 CA103846-07.

\section{REFERENCES}

Amsterdam A, Burgess S, Golling G, Chen W, Sun Z, Townsend K, Farrington S, Haldi M, Hopkins N. 1999. A largescale insertional mutagenesis screen in zebrafish. Genes Dev 13: 2713-2724.

Amsterdam A, Nissen RM, Sun Z, Swindell EC, Farrington S, Hopkins N. 2004a. Identification of 315 genes essential for early zebrafish development. Proc Natl Acad Sci 101: 12792-12797.

Amsterdam A, Sadler KC, Lai K, Farrington S, Bronson RT, Lees JA, Hopkins N. 2004b. Many ribosomal protein genes are cancer genes in zebrafish. PLoS Biol 2: E139.

Armstrong JF, Kaufman MH, Harrison DJ, Clarke AR. 1995. High-frequency developmental abnormalities in p53deficient mice. Curr Biol 5: 931-936.

Arndt CA, Crist WM. 1999. Common musculoskeletal tumors of childhood and adolescence. N Engl J Med 341: 342-352.

Azuma M, Toyama R, Laver E, Dawid IB. 2006. Perturbation of rRNA synthesis in the bap28 mutation leads to apoptosis mediated by $\mathrm{p} 53$ in the zebrafish central nervous system. J Biol Chem 281: 13309-13316.

Bakkers J, Hild M, Kramer C, Furutani-Seiki M, Hammerschmidt M. 2002. Zebrafish DeltaNp63 is a direct target of Bmp signaling and encodes a transcriptional repressor blocking neural specification in the ventral ectoderm. Dev Cell 2: 617-627.

Bartel DP. 2004. MicroRNAs: genomics, biogenesis, mechanism, and function. Cell 116: 281-297.

Berghmans S, Murphey RD, Wienholds E, Neuberg D, Kutok JL, Fletcher CD, Morris JP, Liu TX, Schulte-Merker S, Kanki JP, et al. 2005. p53 mutant zebrafish develop malignant peripheral nerve sheath tumors. Proc Natl Acad Sci 102: 407-412.

Bourdon JC, Fernandes K, Murray-Zmijewski F, Liu G, Diot A, Xirodimas DP, Saville MK, Lane DP. 2005. p53 isoforms can regulate p53 transcriptional activity. Genes Dev 19: $2122-2137$.

Brooks CL, Gu W. 2003. Ubiquitination, phosphorylation and acetylation: the molecular basis for p53 regulation. Curr Opin Cell Biol 15: 164-171.

Brose MS, Volpe P, Feldman M, Kumar M, Rishi I, Gerrero R, Einhorn E, Herlyn M, Minna J, Nicholson A, et al. 2002. BRAF and RAS mutations in human lung cancer and melanoma. Cancer Res 62: 6997-7000.

Caldas H, Holloway MP, Hall BM, Qualman SJ, Altura RA. 2006. Survivin-directed RNA interference cocktail is a potent suppressor of tumour growth in vivo. J Med Genet 43: $119-128$.

Celli J, Duijf P, Hamel BC, Bamshad M, Kramer B, Smits AP, Newbury-Ecob R, Hennekam RC, Van Buggenhout G, van Haeringen A, et al. 1999. Heterozygous germline mutations in the p53 homolog p63 are the cause of EEC syndrome. Cell 99: 143-153.

Ceol CJ, Houvras Y, White RM, Zon LI. 2008. Melanoma biology and the promise of zebrafish. Zebrafish 5: 247-255.

Chakraborty A, Uechi T, Higa S, Torihara H, Kenmochi N. 2009. Loss of ribosomal protein L11 affects zebrafish embryonic development through a p53-dependent apoptotic response. PLoS One 4: e4152.

Chen J, Ng SM, Chang C, Zhang Z, Bourdon JC, Lane DP, Peng J. 2009. p53 isoform delta113p53 is a p53 target gene that antagonizes p53 apoptotic activity via $\mathrm{BclxL}$ activation in zebrafish. Genes Dev 23: 278-290.

Chen J, Ruan H, Ng SM, Gao C, Soo HM, Wu W, Zhang Z, Wen Z, Lane DP, Peng J. 2005. Loss of function of def selectively up-regulates Delta113p53 expression to arrest 
expansion growth of digestive organs in zebrafish. Genes Dev 19: 2900-2911.

Chen Y, Takita J, Hiwatari M, Igarashi T, Hanada R, Kikuchi A, Hongo T, Taki T, Ogasawara M, Shimada A, et al. 2006. Mutations of the PTPN11 and RAS genes in rhabdomyosarcoma and pediatric hematological malignancies. Genes Chromosomes Cancer 45: 583-591.

Cheng R, Ford BL, O'Neal PE, Mathews CZ, Bradford CS, Thongtan T, Barnes DW, Hendricks JD, Bailey GS 1997. Zebrafish (Danio rerio) p53 tumor suppressor gene: $\mathrm{CDNA}$ sequence and expression during embryogenesis. Mol Mar Biol Biotechnol 6: 88-97.

Cichowski K, Shih TS, Schmitt E, Santiago S, Reilly K, McLaughlin ME, Bronson RT, Jacks T. 1999. Mouse models of tumor development in neurofibromatosis type 1. Science 286: 2172-2176.

Danilova N, Sakamoto KM, Lin S. 2008. Ribosomal protein S19 deficiency in zebrafish leads to developmental abnormalities and defective erythropoiesis through activation of p53 protein family. Blood 112: 5228-5237.

Daniotti M, Oggionni M, Ranzani T, Vallacchi V, Campi V, Di Stasi D, Torre GD, Perrone F, Luoni C, Suardi S, et al. 2004. BRAF alterations are associated with complex mutational profiles in malignant melanoma. Oncogene 23: 5968-5977.

Davies H, Bignell GR, Cox C, Stephens P, Edkins S, Clegg S, Teague J, Woffendin H, Garnett MJ, Bottomley W, et al. 2002. Mutations of the BRAF gene in human cancer Nature 417: 949-954.

Donehower LA, Harvey M, Slagle BL, McArthur MJ, Montgomery CA Jr, Butel JS, Bradley A. 1992. Mice deficient for p53 are developmentally normal but susceptible to spontaneous tumours. Nature 356: 215-221.

Ekker SC, Larson JD. 2001. Morphant technology in model developmental systems. Genesis 30: 89-93.

Esquela-Kerscher A, Slack FJ. 2006. Oncomirs microRNAs with a role in cancer. Nat Rev Cancer 6: 259-269.

Golling G, Amsterdam A, Sun Z, Antonelli M, Maldonado E, Chen W, Burgess S, Haldi M, Artzt K, Farrington S et al. 2002. Insertional mutagenesis in zebrafish rapidly identifies genes essential for early vertebrate development. Nat Genet 31: 135-140.

Jacks T, Remington L, Williams BO, Schmitt EM, Halachmi S, Bronson RT, Weinberg RA. 1994. Tumor spectrum analysis in p53-mutant mice. Curr Biol 4: 1-7.

Jackson AL, Linsley PS. 2004. Noise amidst the silence: offtarget effects of siRNAs? Trends Genet 20: 521-524.

Jones SN, Roe AE, Donehower LA, Bradley A. 1995. Rescue of embryonic lethality in Mdm2-deficient mice by absence of p53. Nature 378: 206-208.

Jost CA, Marin MC, Kaelin WG Jr. 1997. p73 is a simian [correction of human] p53-related protein that can induce apoptosis. Nature 389: 191-194.

Kaufmann WK, Nevis KR, Qu P, Ibrahim JG, Zhou T, Zhou Y, Simpson DA, Helms-Deaton J, Cordeiro-Stone M, Moore DT, et al. 2008. Defective cell cycle checkpoint functions in melanoma are associated with altered patterns of gene expression. J Invest Dermatol 128: 175-187.

Kleihues P, Schauble B, zur Hausen A, Esteve J, Ohgaki H. 1997. Tumors associated with p53 germline mutations: a synopsis of 91 families. Am J Pathol 150: 1-13.
Lai K, Amsterdam A, Farrington S, Bronson RT, Hopkins N, Lees JA. 2009. Many ribosomal protein mutations are associated with growth impairment and tumor predisposition in zebrafish. Dev Dyn 238: 76-85.

Langenau DM, Keefe MD, Storer NY, Guyon JR, Kutok JL, Le X, Goessling W, Neuberg DS, Kunkel LM, Zon LI. 2007. Effects of RAS on the genesis of embryonal rhabdomyosarcoma. Genes Dev 21: 1382-1395.

Langheinrich U, Hennen E, Stott G, Vacun G. 2002. Zebrafish as a model organism for the identification and characterization of drugs and genes affecting p 53 signaling. Curr Biol 12: 2023-2028.

Le MT, Teh C, Shyh-Chang N, Xie H, Zhou B, Korzh V, Lodish HF, Lim B. 2009. MicroRNA-125b is a novel negative regulator of p53. Genes Dev 23: 862-876.

Lee H, Kimelman D. 2002. A dominant-negative form of p63 is required for epidermal proliferation in zebrafish. Dev Cell 2: 607-616.

Lee KC, Goh WL, Xu M, Kua N, Lunny D, Wong JS, Coomber D, Vojtesek B, Lane EB, Lane DP. 2008. Detection of the $\mathrm{p} 53$ response in zebrafish embryos using new monoclonal antibodies. Oncogene 27: 629-640.

MacInnes AW, Amsterdam A, Whittaker CA, Hopkins N, Lees JA. 2008. Loss of p53 synthesis in zebrafish tumors with ribosomal protein gene mutations. Proc Natl Acad Sci 105: 10408-10413.

McGrath JA, Duijf PH, Doetsch V, Irvine AD, de Waal R, Vanmolkot KR, Wessagowit V, Kelly A, Atherton DJ, Griffiths WA, et al. 2001. Hay-Wells syndrome is caused by heterozygous missense mutations in the SAM domain of p63. Hum Mol Genet 10: 221-229.

Menon AG, Anderson KM, Riccardi VM, Chung RY, Whaley JM, Yandell DW, Farmer GE, Freiman RN, Lee JK, Li FP, et al. 1990. Chromosome 17p deletions and $\mathrm{p} 53$ gene mutations associated with the formation of malignant neurofibrosarcomas in von Recklinghausen neurofibromatosis. Proc Natl Acad Sci 87: 5435-5439.

Mills AA, Zheng B, Wang XJ, Vogel H, Roop DR, Bradley A. 1999. p63 is a p53 homologue required for limb and epidermal morphogenesis. Nature 398: 708-713.

Montes de Oca Luna R, Wagner DS, Lozano G. 1995. Rescue of early embryonic lethality in mdm2-deficient mice by deletion of p53. Nature 378: 203-206.

Ng JK, Tamura K, Buscher D, Izpisua-Belmonte JC. 1999. Molecular and cellular basis of pattern formation during vertebrate limb development. Curr Top Dev Biol 41: 37-66.

Nichols KE, Malkin D, Garber JE, Fraumeni JF Jr, Li FP. 2001. Germ-line p53 mutations predispose to a wide spectrum of early-onset cancers. Cancer Epidemiol Biomarkers Prev 10: 83-87.

Nielsen GP, Stemmer-Rachamimov AO, Ino Y, Moller MB, Rosenberg AE, Louis DN. 1999. Malignant transformation of neurofibromas in neurofibromatosis 1 is associated with CDKN2A/p16 inactivation. Am J Pathol 155: 1879-1884.

Pan H, Dung HN, Hsu HM, Hsiao KM, Chen LY. 2003. Cloning and developmental expression of p73 cDNA in zebrafish. Biochem Biophys Res Commun 307: 395-400. 
N.Y. Storer and L.I. Zon

Patton EE, Widlund HR, Kutok JL, Kopani KR, Amatruda JF, Murphey RD, Berghmans S, Mayhall EA, Traver D, Fletcher CD, et al. 2005. BRAF mutations are sufficient to promote nevi formation and cooperate with p53 in the genesis of melanoma. Curr Biol 15: 249-254.

Pollock PM, Harper UL, Hansen KS, Yudt LM, Stark M, Robbins CM, Moses TY, Hostetter G, Wagner U, Kakareka J, et al. 2003. High frequency of BRAF mutations in nevi. Nat Genet 33: 19-20.

Pozniak CD, Barnabe-Heider F, Rymar VV, Lee AF, Sadikot AF, Miller FD. 2002. p73 is required for survival and maintenance of CNS neurons. J Neurosci 22: 9800-9809.

Pozniak CD, Radinovic S, Yang A, McKeon F, Kaplan DR, Miller FD. 2000. An anti-apoptotic role for the 553 family member, p73, during developmental neuron death. Science 289: 304-306.

Rentzsch F, Kramer C, Hammerschmidt M. 2003. Specific and conserved roles of TAp73 during zebrafish development. Gene 323: 19-30.

Robu ME, Larson JD, Nasevicius A, Beiraghi S, Brenner C, Farber SA, Ekker SC. 2007. p53 activation by knockdown technologies. PLoS Genet 3: e78.

Rogel A, Popliker M, Webb CG, Oren M. 1985. p53 cellular tumor antigen: analysis of mRNA levels in normal adult tissues, embryos, and tumors. Mol Cell Biol 5: 28512855.

Romani M, Tonini GP, Banelli B, Allemanni G, Mazzocco K, Scaruffi P, Boni L, Ponzoni M, Pagnan G, Raffaghello L, et al. 2003. Biological and clinical role of p73 in neuroblastoma. Cancer Lett 197: 111-117.

Sah VP, Attardi LD, Mulligan GJ, Williams BO, Bronson RT, Jacks T. 1995. A subset of p53-deficient embryos exhibit exencephaly. Nat Genet 10: 175-180.

Scacheri PC, Rozenblatt-Rosen O, Caplen NJ, Wolfsberg TG, Umayam L, Lee JC, Hughes CM, Shanmugam KS, Bhattacharjee A, Meyerson M, et al. 2004. Short interfering RNAs can induce unexpected and divergent changes in the levels of untargeted proteins in mammalian cells. Proc Natl Acad Sci 101: 1892-1897.

Schmid P, Lorenz A, Hameister H, Montenarh M. 1991. Expression of p53 during mouse embryogenesis. Development 113: 857-865.
Sinha AU, Kaimal V, Chen J, Jegga AG. 2008. Dissecting microregulation of a master regulatory network. $B M C$ Genomics 9: 88.

Skarie JM, Link BA. 2008. The primary open-angle glaucoma gene WDR36 functions in ribosomal RNA processing and interacts with the p53 stress-response pathway. Hum Mol Genet 17: 2474-2485.

Stratton MR, Fisher C, Gusterson BA, Cooper CS. 1989. Detection of point mutations in $\mathrm{N}$-ras and $\mathrm{K}$-ras genes of human embryonal rhabdomyosarcomas using oligonucleotide probes and the polymerase chain reaction. Cancer Res 49: 6324-6327.

Thisse C, Neel H, Thisse B, Daujat S, Piette J. 2000. The $\mathrm{Mdm} 2$ gene of zebrafish (Danio rerio): preferential expression during development of neural and muscular tissues, and absence of tumor formation after overexpression of its cDNA during early embryogenesis. Differentiation 66: 61-70.

Tuveson DA, Weber BL, Herlyn M. 2003. BRAF as a potential therapeutic target in melanoma and other malignancies. Cancer Cell 4: 95-98.

Vogel KS, Klesse LJ, Velasco-Miguel S, Meyers K, Rushing EJ, Parada LF. 1999. Mouse tumor model for neurofibromatosis type 1. Science 286: 2176-2179.

Vogelstein B, Lane D, Levine AJ. 2000. Surfing the p53 network. Nature 408: 307-310.

Xia SJ, Pressey JG, Barr FG. 2002. Molecular pathogenesis of rhabdomyosarcoma. Cancer Biol Ther 1: 97-104.

Yang A, Kaghad M, Wang Y, Gillett E, Fleming MD, Dotsch V, Andrews NC, Caput D, McKeon F. 1998. p63, a p53 homolog at $3 \mathrm{q} 27-29$, encodes multiple products with transactivating, death-inducing, and dominant-negative activities. Mol Cell 2: 305-316.

Yang A, Schweitzer R, Sun D, Kaghad M, Walker N, Bronson RT, Tabin C, Sharpe A, Caput D, Crum C, et al. 1999. p63 is essential for regenerative proliferation in limb, craniofacial and epithelial development. Nature 398: 714-718.

Yang A, Walker N, Bronson R, Kaghad M, Oosterwegel M, Bonnin J, Vagner C, Bonnet H, Dikkes P, Sharpe A, et al. 2000. p73-deficient mice have neurological, pheromonal and inflammatory defects but lack spontaneous tumours. Nature 404: 99-103. 


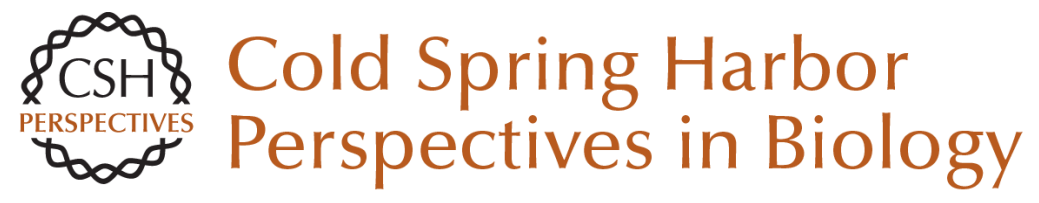

\section{Zebrafish Models of p53 Functions}

Narie Y. Storer and Leonard I. Zon

Cold Spring Harb Perspect Biol 2010; doi: 10.1101/cshperspect.a001123 originally published online May 5,2010

\section{Subject Collection The p53 Family}

The Origins and Evolution of the p53 Family of Genes

Vladimir A. Belyi, Prashanth Ak, Elke Markert, et al.

Mouse Models of p53 Functions

Guillermina Lozano

TP53 Mutations in Human Cancers: Origins,

Consequences, and Clinical Use

Magali Olivier, Monica Hollstein and Pierre Hainaut

p53 Research: The Past Thirty Years and the Next

Thirty Years

David Lane and Arnold Levine

Transcriptional Regulation by P53

Rachel Beckerman and Carol Prives

p53-based Cancer Therapy

David P. Lane, Chit Fang Cheok and Sonia Lain

Phylogeny and Function of the Invertebrate p53

Superfamily

Rachael Rutkowski, Kay Hofmann and Anton Gartner

Tied Up in Loops: Positive and Negative

Autoregulation of p53

Xin Lu
The Tumor Suppressor p53: From Structures to

Drug Discovery

Andreas C. Joerger and Alan R. Fersht

p53 Regulation of Metabolic Pathways

Eyal Gottlieb and Karen H. Vousden

The Regulation of the p53-mediated Stress

Response by MDM2 and MDM4 Mary Ellen Perry

Zebrafish Models of p53 Functions Narie Y. Storer and Leonard I. Zon

$p 63$ and p73, the Ancestors of p53

V. Dötsch, F. Bernassola, D. Coutandin, et al.

Pathologies Associated with the p53 Response Andrei V. Gudkov and Elena A. Komarova

Single-nucleotide Polymorphisms in the p53

Signaling Pathway

Lukasz F. Grochola, Jorge Zeron-Medina, Sophie Mériaux, et al.

Clinical Outcomes and Correlates of TP53

Mutations and Cancer

Ana I. Robles and Curtis C. Harris

For additional articles in this collection, see http://cshperspectives.cshlp.org/cgi/collection/

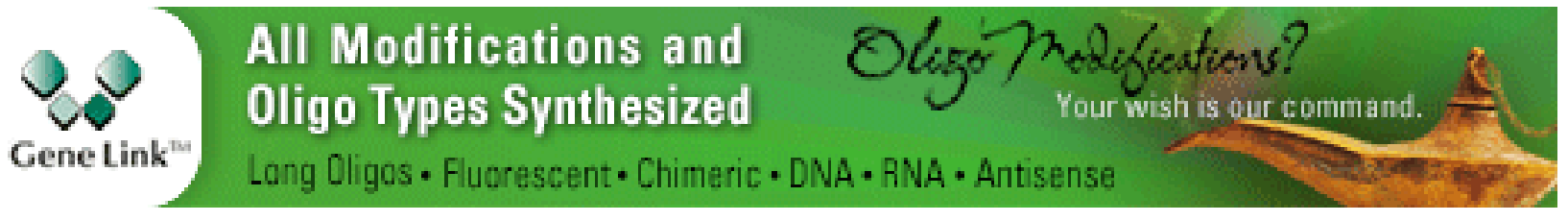

Copyright @ 2010 Cold Spring Harbor Laboratory Press; all rights reserved 